\title{
APLICAÇÃO DA TEORIA DAS FILAS PARA AVALIAÇÃO DO DESEMPENHO E MELHORAMENTO NO SERVIÇO DE ENTREGA EM UMA MICROEMPRESA DE DISTRIBUIÇÃO DE ÁGUA E GÁS
}

\author{
João Mario Rufino da Silva \\ Universidade Federal de Campina Grande \\ Rua Luiz Grande, S/N - CEP 58540-000 - Sumé - Paraíba - Brasil \\ joaomario1997@hotmail.com \\ Daniel Augusto de Moura Pereira \\ Universidade Federal de Campina Grande \\ Rua Luiz Grande, S/N - CEP 58540-000 - Sumé - Paraíba - Brasil \\ danielmoura@ufcg.edu.br \\ Antônio Carlos da Silva Batista Vaz \\ Universidade Federal de Mato Grosso \\ a.carlosvaz1@gmail.com
}

\section{RESUMO}

Com a constante mudança no setor de serviços quando se trata de atendimento ao cliente, é necessário um maior aperfeiçoamento e maior investimento na qualidade da prestação do mesmo. Neste cenário, a busca pelo menor tempo e maior eficácia no atendimento, tem feito com que os empresários invistam mais na qualidade do serviço, fazendo com que os clientes se sintam satisfeitos e não sejam forçados a buscarem melhorias na concorrência. Desta forma se torna imprescindível o uso de ferramentas que possam auxiliar este processo. A elaboração deste estudo consiste na utilização das medidas de desempenho do sistema a partir da Teoria das Filas, para visualização da eficiência do sistema quanto ao atendimento em uma distribuidora de Água e Gás

Palavra-chave: Teoria das Filas; Medidas de Desempenho.

\begin{abstract}
With the constant change in the service sector when it comes to customer service, it is necessary to improve and invest more in the quality of service. In this scenario, the search for shorter time and more effective service has made entrepreneurs invest more in quality of service, making customers feel satisfied and not forced to seek improvements in competition. In this way it becomes essential to use tools that can help this process. The elaboration of this study consists in the use of the performance measures of the system from the Theory of Queues, to visualize the efficiency of the system as to the attendance at a Water and Gas distributor.
\end{abstract}

Keywords: Queue Theory; Performance Measures. 


\section{Como Citar:}

SOBRENOME, Nome Autor 1; SOBRENOME, Nome Autor 2. Título do Trabalho. In: SIMPÓSIO DE PESQUISA OPERACIONAL E LOGÍSTICA DA MARINHA, 19., 2019, Rio de Janeiro, RJ. Anais [...]. Rio de Janeiro: Centro de Análises de Sistemas Navais, 2019.

\section{INTRODUÇÃO}

O setor brasileiro de prestação de serviços é um dos que vem mudando constantemente quando se fala em atendimento de pessoas. Para Marquez (2018), os clientes tornaram-se cada vez mais exigentes com relação ao atendimento, principalmente quando se trata do tempo, ou seja, querem um serviço de forma rápida e confortável, e atualmente os setores de prestação de serviço trabalham para promover isso para os clientes e fazer com que eles não sejam forçados a buscar melhorias na concorrência. Isso é fruto das exigências e dos diversos perfis dos usuários, onde demanda um aperfeiçoamento e um maior investimento em qualidade no atendimento voltado para um conforto maior para os clientes.

Neste sentido, um dos problemas no setor de distribuição (entrega) convencional de água e gás é a demora, decorrente de outros problemas como, falta de organização dos pedidos, dificuldade de comunicação entre o atendente e o cliente, dificuldade de comunicação entre o atendente e o entregador, ou seja, há problemas em repassar as informações fornecidas pelo cliente. Além disso, ainda se nota que pode ocorrer à inversão da ordem dos pedidos por motivo de distância, e diversos outros problemas que afetam diretamente o cliente.

$\mathrm{O}$ atendimento em uma distribuidora de água e gás de uma cidade do interior da Paraíba apresenta falhas, sobretudo no que tange a satisfação do consumidor, pois é notada uma superlotação no atendimento, principalmente em dias de grande fluxo de pedidos, normalmente primeiros dias do mês, os dias da feira livre os finais de semana.

Portanto, o objetivo deste trabalho é avaliar o sistema de filas formadas para verificar onde se encontram os possíveis déficits como consequência da ineficiência no atendimento do local de estudo supracitado.

\section{FUNDAMENTAÇÃO TEÓRICA}

\subsection{SimulaÇão}


Para Camelo et al. (2010), a simulação é definida como uma técnica do ramo da Pesquisa Operacional que possibilita a imitação do funcionamento do sistema real, ou seja simular significa reproduzir o funcionamento de determinado sistema.

Já para Silva (1998), simular significa reproduzir o funcionamento de um sistema, com a ajuda de determinado modelo que permita testar algumas hipóteses sobre o valor das variáveis a serem controladas, e com a definição dessas variáveis é possível melhorar o sistema estudado.

Conforme explica Camelo (2010), a simulação é um instrumento poderoso para analisar e determinar deficiências no sistema, permitindo quantificar o desempenho do mesmo, aplicando assim possíveis melhorias.

\subsection{Teoria das Filas}

Fila é o efeito de certa ineficiência entre a demanda dos usuários e a capacidade do serviço oferecido, mas, Moreira (2010) argumenta que nem sempre a formação de fila está associada à capacidade de atendimento, mas também à variabilidade tanto no intervalo entre chegadas como no tempo de atendimento. A formação das filas pode ser vistas em qualquer atividade, por mais simples que ela seja, como, supermercados, bancos, lanchonetes, lojas ou até mesmo em linhas de produção onde requer a espera por determinados produtos.

Miranda et al. (2006) apresentam a Teoria das Filas como sendo um conjunto de teoremas, que abrangem cálculos probabilísticos, que visam explicar o comportamento de atendimento a serviços enfileirados, sendo que, o estudo de Teoria de Filas trata o fenômeno de aguardar em fila usando medidas representativas da performance do sistema, tais como comprimento médio da fila, tempo médio de espera na fila entre outros. $\mathrm{O}$ estudo de Teoria das Filas pertence a área de pesquisa operacional, estando também ligada aos processos estocásticos.

Segundo Camelo et al. (2010), os principais elementos de uma fila são: cliente, fila e canal de atendimento, como está representado na figura 1. 
Figura 1 - Elementos de uma Fila

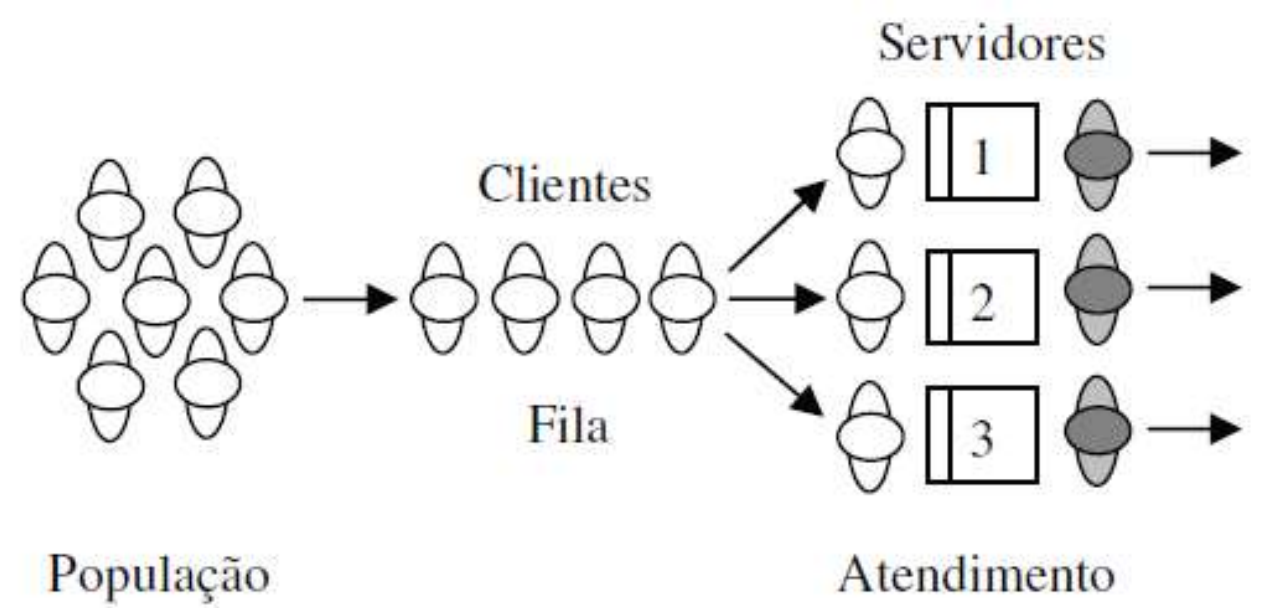

Fonte: Camelo et al. (2010)

\subsubsection{Objetivo da Teoria das Filas}

Segundo Moreira (2010), objetivo da teoria das filas é fornecer dados que ajudem a modelar a prestação de serviço do sistema e buscar um equilíbrio entre os custos para prestação daquele serviço e custos com atraso devido à ineficiência do mesmo (Formação das Filas).

Em atividades como a produção de itens que exigem o carregamento de materiais, sejam manualmente ou em máquinas, a espera destes materiais para produção pode significar custos. Esse tempo de espera pode ser maior ou menor de acordo com a capacidade do carregamento, mas os custos desta capacidade geralmente também são altos, a análise com base na teoria das filas pode influenciar para devidas tomadas de decisão que podem minimizar esses custos.

\section{METODOLOGIA}

\subsection{Pesquisa}

De acordo com Lakatos e Marconi (2006), pesquisa é uma atividade voltada à busca de respostas e à solução de problemas para questões propostas, para isso utilizam-se métodos científicos.

Para Gil (2008), a pesquisa pode ser pura ou aplicada. A pesquisa pura procura desenvolver progresso da ciência, alargando os conhecimentos científicos sem se preocupar 
diretamente com suas aplicações e consequências práticas. Já a pesquisa aplicada, proporciona várias interfaces com a pesquisa pura, dependendo de suas descobertas e do enriquecimento do seu incremento, sua principal característica está na aplicação, utilização e seus conhecimentos na prática.

Neste trabalho foi feito uso da pesquisa aplicada, uma vez que foram utilizados dados obtidos em campo com a aplicação dos conhecimentos técnicos de teoria das filas e ferramentas de gestão da qualidade.

O estudo ainda pode ser classificado como pesquisa quantitativa, uma vez que se trata da coleta e inferência dos tempos de atendimento em uma distribuidora de água e gás, como também medidas de desempenho da fila em estudo.

\subsection{Metodologia de Coleta e Inferência de Dados}

O presente estudo foi elaborado através de visitas in loco no serviço de entrega em uma distribuidora de água e gás na cidade de Sumé-PB. Para coleta dos tempos de entrada na fila e de atendimento, utilizou-se um cronometro digital. O processo de recolhimento de dados durou cinco dias aleatórios em diferentes turnos. Nesse período foram registrados 135 tempos de atendimento.

De acordo com Chwif e Medina (2010), o tamanho da amostra deve estar entre 100 e 200 observações; amostras com menos de 100 observações podem comprometer a identificação do melhor modelo probabilístico, e amostra com mais de 200 observações não trazem ganhos significativos ao estudo.

Após a coleta, foi realizado o registro dos tempos em uma planilha no Microsoft Excel para assim, obter em minutos o intervalo de chegada entre pedidos e o tempo de duração do atendimento. Com a finalidade de se fazer uma análise mais detalhada e apurada dos dados, foi utilizado o software Minitab versão 18, gerando a estatística descritiva, gráficos de dispersão e histogramas, para assim, observar o comportamento de todo o sistema no período estudado.

Por meio da inferência dos dados tornou-se possível calcular as medidas de desempenho do sistema, ou seja, as medidas da fila no sistema de entrega. Logo após esses cálculos, ficaram visíveis as deficiências no atendimento da distribuidora, diante disto se fez 
Figura 2: Gráfico Metodológico

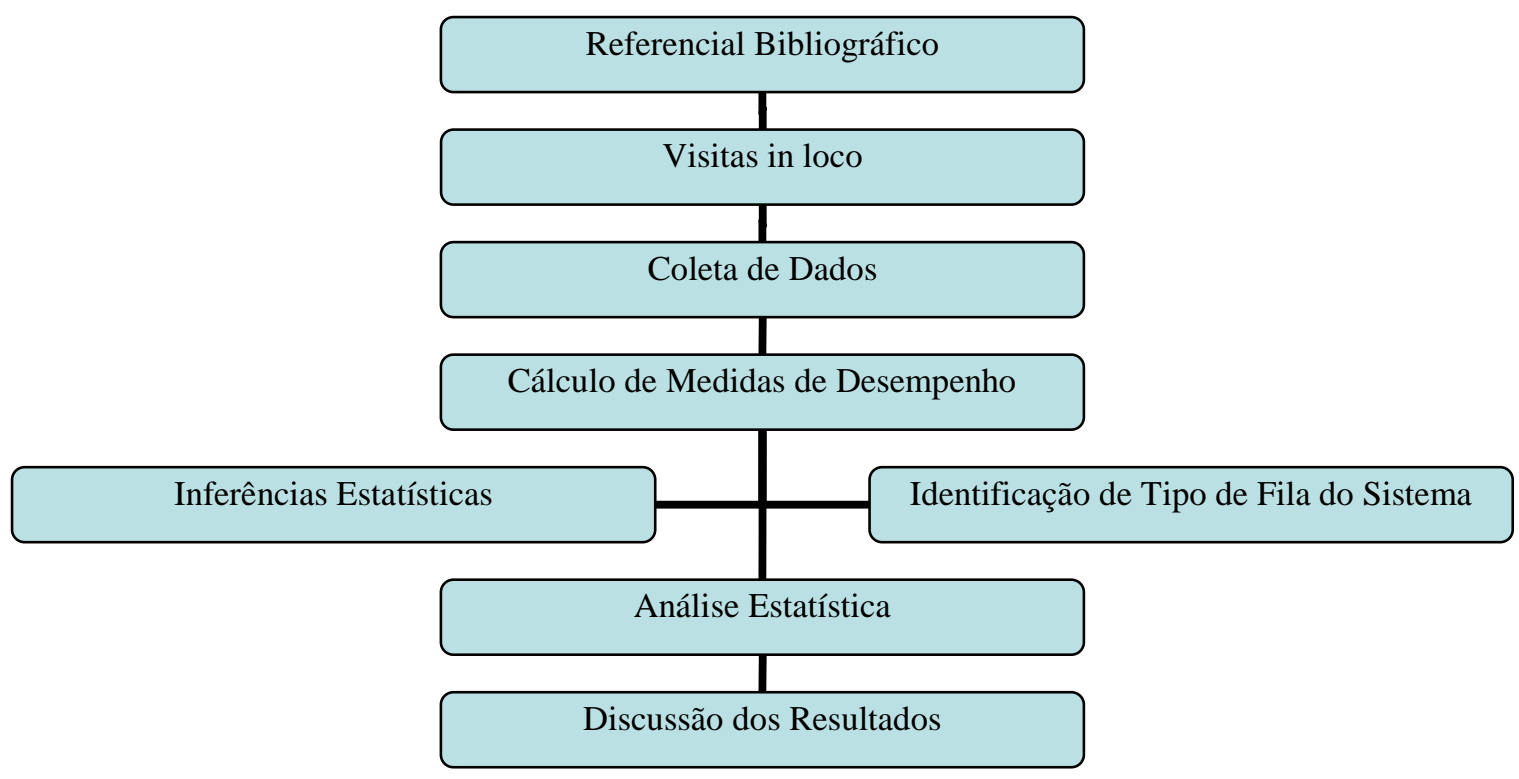

Fonte: Os autores (2018)

\section{RESULTADOS}

\subsection{Tratamento dos Dados}

Inicialmente verificou-se a existência de possíveis outliers na amostra. Após a remoção de 8 (oito) outliers (que são pontos extremos, muito altos ou muito baixos, que podem comprometer o estudo), foi realizado o levantamento estatístico descritivo conforme mostra a figura 3. 
Figura 3: Estatística Descritiva para o intervalo de tempo entre os pedidos.

$\begin{array}{lc}\text { Variável } & \begin{array}{c}\text { Intervalo entr } \\ \text { pedidos (min. }\end{array} \\ \text { Média } & 5,495 \\ \text { Desvio Padrão } & 4,945 \\ \text { Variância } & 24,449 \\ \text { Coeficiente de Variação } & 89,99 \\ \text { Mínimo } & 0,000 \\ \text { Primeiro Quartil (Q1) } & 1,433 \\ \text { Mediana } & 4,333 \\ \text { Terceiro Quartil (Q3) } & 8617 \\ \text { Máximo } & 22,583 \\ \text { Amplitude } & 22,583 \\ \text { Moda } & 0 \\ \text { Número de Moda } & 6 \\ \text { Assimetria } & 1,17\end{array}$

Fonte: Os autores (2018)

De acordo com análise extraída do software Minitab, a cada 5, 495 minutos, em média, um pedido entra na fila, com um desvio padrão de 4, 945 minutos entre os dados obtidos.

Com o intuito de mostrar a influência que uma variável tem sobre a outra, foi construído o gráfico de dispersão do intervalo entre os pedidos, como mostra a figura 4.

Figura 4 - Gráfico de dispersão intervalo entre pedidos

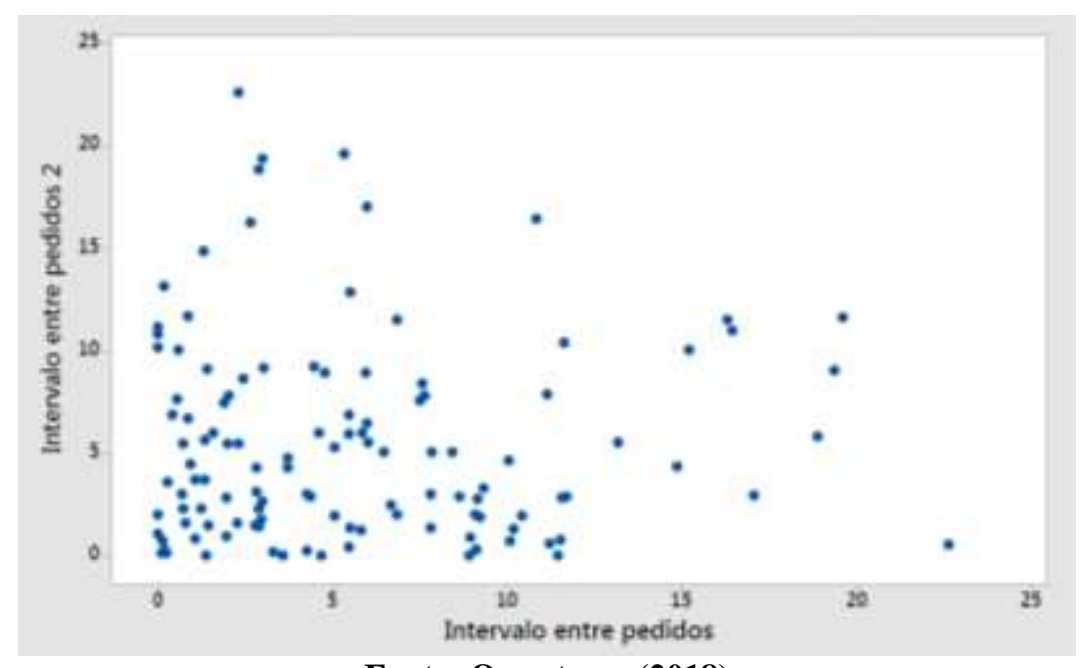

Fonte: Os autores (2018) 
Da figura 4 poder-se perceber que não existe nenhuma relação entre a entrada do processo (x) e a saída do processo (y), uma vez que os pontos plotados não seguem nenhum padrão. Complementarmente, objetivando verificar os dados através de uma análise quantitativa e um agrupamento de classes, formou-se um gráfico do tipo histograma, mostrado na figura 5, permitindo outro tipo de estudo das informações coletadas.

Figura 5 - Histograma dos tempos de chegada dos clientes

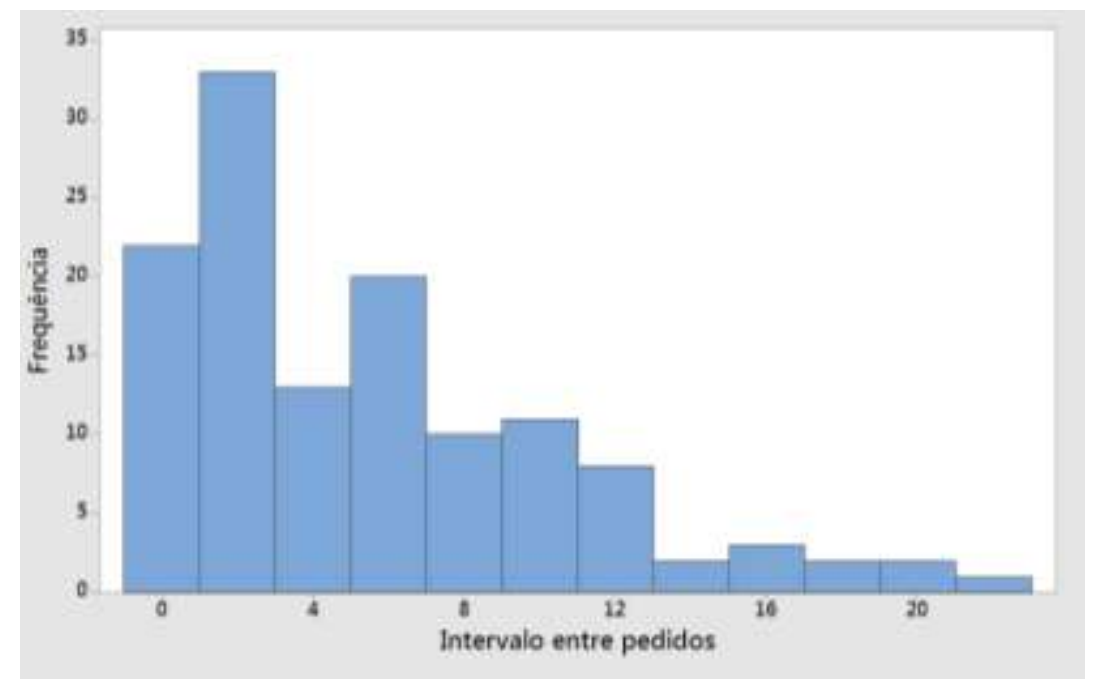

Fonte: Os autores (2018)

Da figura 5 pode-se ver que os dados estão concentrados em sua maioria entre 0 e 8 minutos, representando cerca de $72,6 \%$ da amostra. Este levantamento evidencia o comportamento de uma distribuição contínua exponencial, pois os dados resultam na contagem de eventos; além do que, todos os eventos representam tempos de chegadas aleatórios característicos a esse tipo de distribuição.

Foi realizado um teste de aderência dos dados de intervalo entre os pedidos, conforme ilustra a figura 6. Para este estudo do valor-P, foi analisada a tabela de probabilidade, onde foi visto este valor para o índice de confiança de 95\%, ou seja, o nível de significância é de 5\%. E caso o valor-P seja maior que 0,05 , os dados aderem a distribuição que foi mencionada no teste. Sabendo que o intervalo de confiança é de $95 \%$, foi encontrado o valor-P de 0,343, o qual é maior que o nível de significância sendo de 0,05, logo a hipótese nula é aceita, ou seja, os dados realmente aderem à distribuição exponencial. 
Figura 6 - Teste de aderência dos dados de intervalo entre pedidos

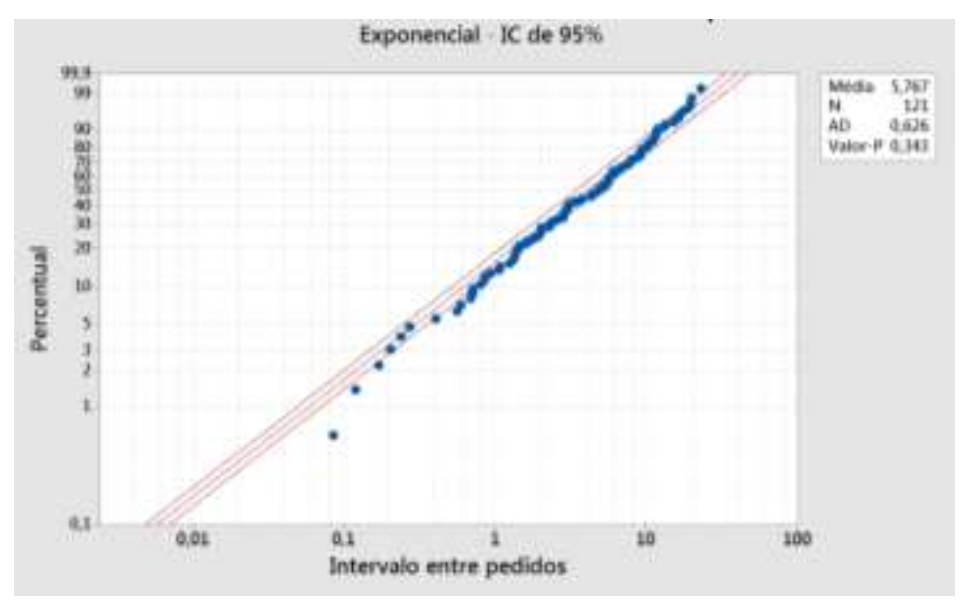

Fonte: Os autores (2018)

De maneira análoga, realizou o mesmo procedimento estatístico para os dados de atendimento do local estudo. Foram encontrados 7 (sete) outliers que, por conseguinte, foram eliminados. A partir dai realizou-se a estatística descritiva dos dados, conforme ilustra a figura 7.

Figura 7 - Estatística Descritiva para o intervalo de tempo de atendimento.

\begin{tabular}{l|c}
\hline \multicolumn{1}{c|}{ Variável } & $\begin{array}{c}\text { Tempo de } \\
\text { atendimento (min.) }\end{array}$ \\
\hline Média & 26,77 \\
\hline Desvio Padrão & 12,01 \\
\hline Variância & 144,20 \\
\hline Coeficiente de Variação & 44,86 \\
\hline Mínimo & 2,80 \\
\hline Primeiro Quartil (Q1) & 17,65 \\
\hline Mediana & 26,38 \\
\hline Terceiro Quartil (Q3) & 33,52 \\
\hline Máximo & 58,13 \\
\hline Amplitude & 55,33 \\
\hline Moda & 31,$93 ; 33,51$ \\
\hline Número de Moda & 2 \\
\hline Assimetria & 0,36 \\
\hline
\end{tabular}

Fonte: Os autores (2018)

A partir das informações extraída do software Minitab, constatou-se que se um pedido for realizado, ele demorará, em média, 26,77 minutos para ser concluído. Dessa forma, levando em consideração que o pedido leva cerca de 5,495 minutos para entrar na fila, isso explica a formação das mesmas, uma vez que quando um pedido é dado por completo, já existem mais quatro em espera.

Para melhor ilustrar a correlação nesse compilado de dados, foi montado um diagrama de dispersão do tempo de atendimento, conforme está apresentado na Figura 8. 
Figura 8 - Gráfico de dispersão tempo de atendimento

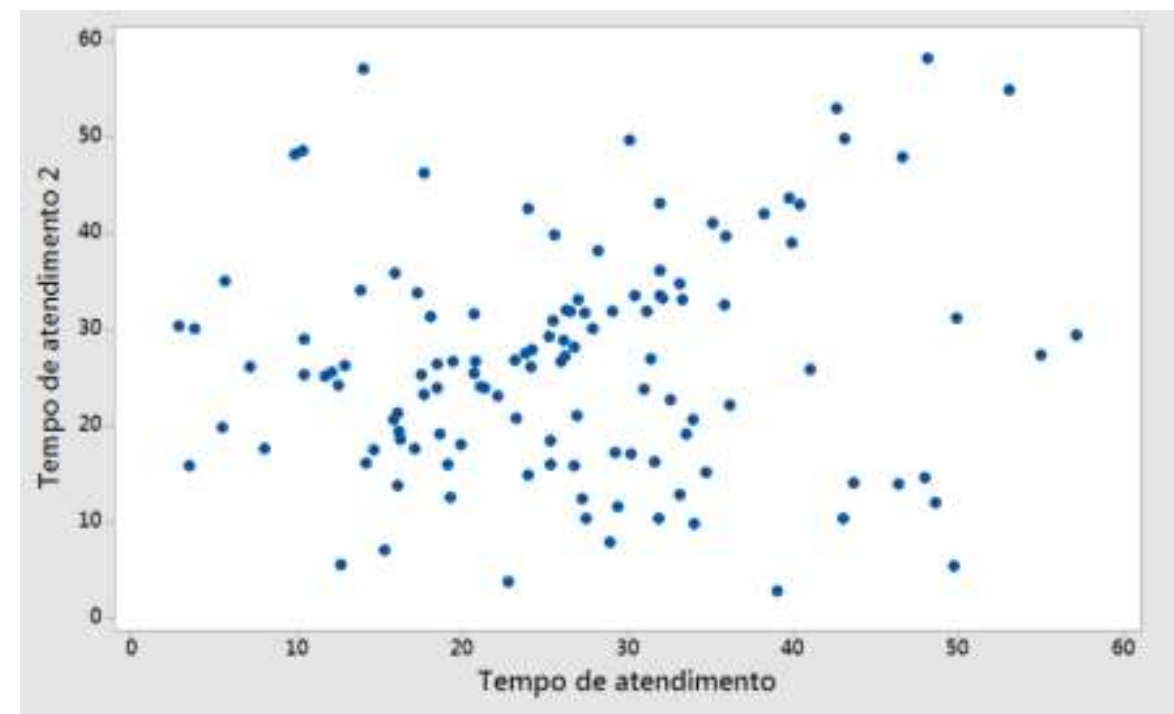

Fonte: Os autores (2018)

Como se pode observar, não existe relação entre a entrada do processo (x) e a saída do processo (y) - uma vez que os pontos plotados são aleatórios. Complementarmente, objetivando verificar os dados através de uma análise quantitativa e um agrupamento de classes, formou-se um gráfico do tipo histograma, mostrado na figura 9, permitindo outro tipo de estudo das informações coletadas.

Figura 9 - Histograma dos tempos de atendimento

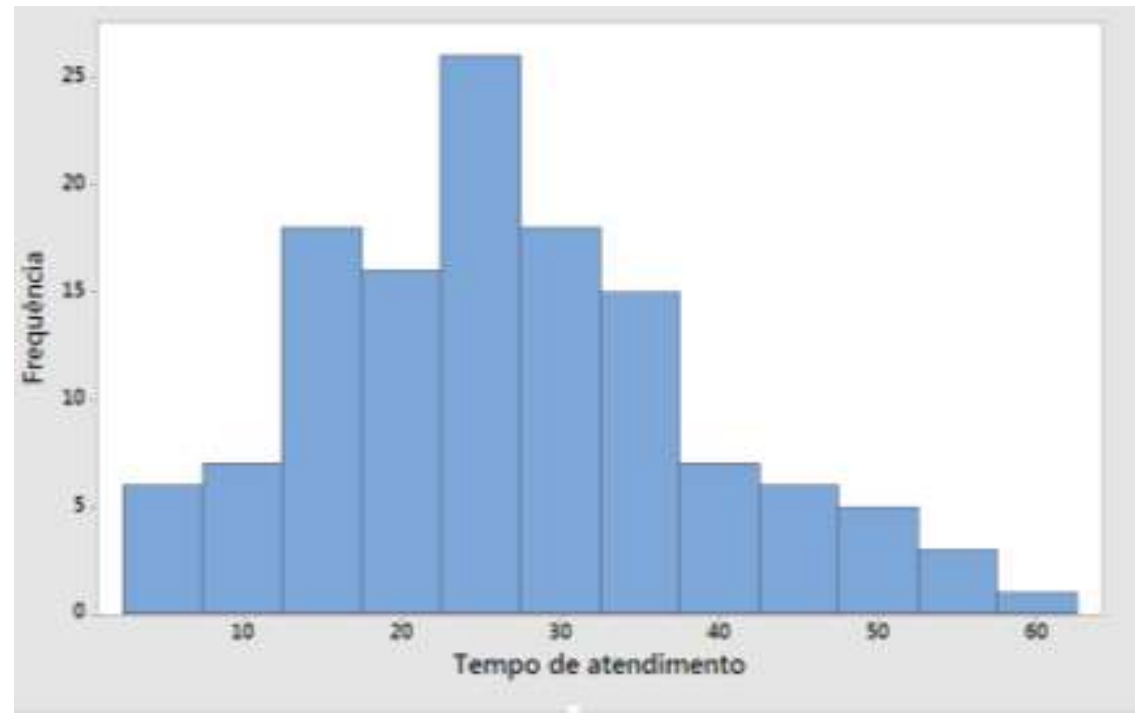

Fonte: Os autores (2018)

Como se pode perceber na figura 9, os dados mostram que a duração do atendimento em sua maioria estão entre 15 e 35 minutos, representando cerca de $68,15 \%$ da amostra. A figura 9 
também apresenta o baixo percentual de atendimentos efetuados com rapidez, compreendidos entre 0 e 10 minutos, que representam $8,15 \%$ dos pedidos registrados.

Para verificar se o conjunto de dados do tempo de atendimento pode ter sido gerado a partir de uma distribuição de probabilidade específica, foi feito um teste de aderência, apresentado na Figura 10.

Figura 10 - Teste de aderência dos dados

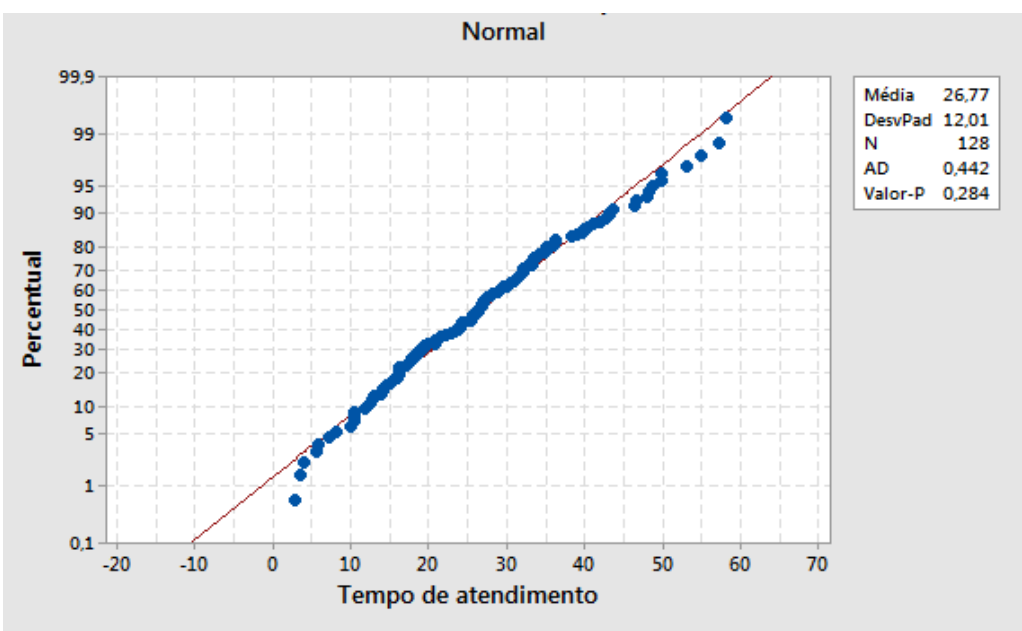

Fonte: Os autores (2018)

Para este estudo do valor-P, foi analisada a tabela de probabilidade, onde foi visto este valor para o índice de confiança de $95 \%$, ou seja, o nível de significância é de 5\%. Compreendendo que o valor-P encontrado é maior que o nível de significância de $5 \%$, a hipótese nula é aceita, assim os dados seguem a o comportamento da distribuição normal.

\subsection{Medidas de Sistema}

A média de intervalos dos pedidos foi a cada 5, 495 minutos, e os dados tratados com a Distribuição Exponencial, segue a seguinte fórmula:

$$
\begin{gathered}
\lambda=1 / \text { média de tempo de chegada } \\
\lambda=1 / 5,495 \\
\lambda=0,182 / \mathrm{min}
\end{gathered}
$$

A média de duração do tempo de atendimento foi 16,77 minutos, e os dados tratados com a Distribuição Exponencial, segue a seguinte fórmula:

$$
\mu=1 / \text { média de tempo }
$$




$$
\begin{gathered}
\mu=1 / 26,77 \\
\mu=0,037 \text { atendimentos } / \text { min }
\end{gathered}
$$

Para o sistema em estudo, onde existem mais de um servidor, é necessário que a taxa de atendimento seja a taxa do conjunto do sistema, ou seja, será feita a multiplicação por 2 , pois existem 2 entregadores na empresa. Logo a taxa de atendimento do conjunto será:

$$
\begin{gathered}
\mu=0,037 \mathrm{x} 2 \\
\mu=0,074 \text { atendimentos } / \mathrm{min}
\end{gathered}
$$

A mesma foi obtida pela divisão do ritmo de chegada dos pedidos ( $\lambda$ ) pela multiplicação entre o número de servidores e o ritmo médio de atendimento $(c \mu)$, que foram encontrados através da análise estatística na inferência dos dados, exceto o valor do ritmo médio de atendimento " $\mu$ " que foi adotado por definição da razão de uma hora pelo tempo de atendimento: $\mu=2 * \frac{1}{26,77}{ }^{*}$, assim o valor aproximado de $\mu$ é de 0,074 atendimentos/min. Dessa forma o valor da taxa de utilização é de aproximadamente 1,23.

$$
\begin{gathered}
\rho=\frac{\lambda}{c \mu} \\
\rho=\frac{0,182}{2 \times 0,074} \\
\rho=1,23 \quad \rho=1,23 \times 100=123 \%
\end{gathered}
$$

Denotando a taxa média de chegada dos clientes na fila como $\lambda$ e a taxa média de atendimento como $\mu$, encontramos a probabilidade que o sistema esteja ocupado, nesse caso quando $\rho>1(\lambda>\mathrm{c} \mu)$, em que $\mathrm{c}$ é a quantidade de servidores, o número de chegadas ao sistema excede a taxa média de serviço $(0,182>0,074)$, ou seja, quando o tempo avança a fila se tornará cada vez maior, impedindo um possível equilíbrio no sistema e ocasionando grandes filas. Para o sistema ser classificado como equilibrado, seria necessário que $\rho<1$.

O numero médio de clientes na fila de espera trata-se somente dos clientes que estão aguardando, ou seja, não conta os pedidos que já estão sendo entregues.

$$
\begin{gathered}
\mathrm{NF}=\lambda^{2} /(\mu(\lambda-\mu)) \\
\mathrm{NF}=0.182^{2} /(0,074 \times(0,182-0,074))
\end{gathered}
$$




$$
\mathrm{NF}=4,15
$$

O número médio de clientes no sistema trata-se de todos os pedidos, ou seja, os que estão na fila e os que já estão sendo entregues.

$$
\begin{gathered}
\mathrm{N}=\mathrm{NF}+(\lambda / \mu) \\
\mathrm{N}=6,17+(0,182 / 0,074) \\
\mathrm{N}=6,59
\end{gathered}
$$

O tempo médio que o cliente espera na fila aborda o tempo desde a ligação até o momento em que o pedido sai para ser entregue.

$$
\begin{gathered}
\mathrm{TF}=\mathrm{NF} / \lambda \\
\mathrm{TF}=4,15 / 0,182 \\
\mathrm{TF}=22,8 \mathrm{~min}
\end{gathered}
$$

O tempo médio que o cliente espera no sistema aborda o tempo desde a ligação até o pedido ser entregue no local desejado.

$$
\begin{gathered}
\mathrm{T}=\mathrm{T} / \lambda \\
\mathrm{T}=6,59 / 0,182 \\
\mathrm{~T}=36,20 \mathrm{~min}
\end{gathered}
$$

Verificou-se pelos resultados apresentados, que a taxa de chegada de clientes na fila é de 0,182 pedidos por minuto, equivalendo à chegada de 1 cliente a cada 5 minutos e meio aproximadamente, vale ressaltar que isso varia, pois nos dias de coleta de dados houve casos que chegaram até 5 pedidos nesse mesmo intervalo de tempo, dai o elevado número de clientes na fila de espera. Percebeu-se também que a taxa de atendimento do conjunto de servidores da empresa é de 0,074 clientes por minuto, dai podemos notar a ineficiência no sistema, uma vez que a taxa de atendimento é muito menor que a taxa de chegada, isso justifica a probabilidade alta que o sistema esteja ocupado.

A probabilidade que o sistema esteja ocupado é de $123 \%$, e em média possui 4 clientes na fila de espera e 6 clientes no sistema. O tempo médio que cada cliente espera na fila (espera desde a ligação até a saída do entregador) é 22,8 minutos, ou seja, 22 minutos e 48 segundos. E o usuário espera em média 36 minutos e 12 segundos no sistema (tempo de espera desde a ligação até a conclusão da entrega). 
Logo, percebe-se que mesmo com um número considerável de entregadores ainda há um número elevado de clientes esperando na fila, assim como o cliente espera muito tempo para que seu pedido seja entregue.

\section{CONCLUSÃO}

O objeto de estudo deste trabalho foi a aplicação da teoria das filas em uma distribuidora de água e gás. $\mathrm{O}$ atendimento não apresentava resultados satisfatórios, pois mostrava altos tempos na efetuação da entrega. A falta de qualidade na prestação do serviço acaba gerando a insatisfação do cliente, uma vez que este espera muito tempo para que seu pedido seja entregue, problema este que pode comprometer significativamente as vendas da empresa.

As medidas de desempenho do sistema possibilitaram a percepção das taxas de atendimento, de chegada dos clientes e utilização do sistema. Possibilitou ainda cálculos do tamanho da fila, número de clientes no sistema e ainda os tempos de espera. Viu-se também que a empresa em análise apresenta alguns problemas, tais como: ordem de entregas indefinidas, sobrecarga de atividades para alguns funcionários, além da adaptação dos mesmos para com novos métodos de entregas, como o reboque nas motos.

Uma vez que o principal fator formador de filas durante o estudo se deu quando os trabalhadores foram alocados para atividades como: descarga de caminhões; remoção dos rótulos dos vasilhames e etc. esse acontecimento teve um grande impacto, pois diminuiu o número de entregadores disponíveis, na maioria das vezes o serviço de entrega ficava parado, pois os dois funcionários responsáveis eram chamados para descarregar os caminhões. Aumentando assim a fila de pedidos e consequentemente o tempo de atendimento aos clientes.

\section{REFERÊNCIAS BIBLIOGRÁFICAS}

CAMELO, Gustavo Rossa et al. teoria das filas e da simulação aplicada ao embarque de minério de ferro e manganês no terminal marítimo de ponta da madeira. 2010.

Disponível em: <http://novo.more.ufsc.br/homepage/inserir_homepage>. Acesso em: 15 nov. 2018.

CHIWF, L; MEDINA, A. C. Modelagem e simulação de eventos discretos: Teoria \& aplicações. 3. Ed. São Paulo: Ed. do autor, 2010. 
GIL, Antonio Carlos. Como elaborar projetos de pesquisa. 4. ed. São Paulo: Atlas, 2008. MARCONI, M. de A.; LAKATOS, E. M. Fundamentos de Metodologia Científica. $6^{\text {a }}$. ed. São Paulo: Atlas, 2006. 315p.

MARQUEZ, Gabriel. Qual a importância de monitorar os concorrentes? Disponível em: $<$ https://conube.com.br/blog/qual-a-importancia-de-monitorar-os-concorrentes/>. Acesso em: 03 out. 2018.

MIRANDA, Juliano Coelho et al. O Software ARENA. Monografia. Centro Universitário do Sul de Minas - UNIS-MG, Varginha - MG, 2006.

MOREIRA, D. A. Pesquisa Operacional: Curso Introdutório. $3^{\text {a }}$.ed. ver. eatu. São Paulo: Cengage Learning, 2010. 356 p.

SILVA, E.M. et al. Pesquisa Operacional. Para cursos de: economia, administração e ciências contábeis. São Paulo: Atlas, 1998.

As Referências Bibliográficas devem seguir as normas da ABNT/NBR 6023. 